$\Rightarrow$ RHEUMATOID ARTHRITIS

\section{Upadacitinib in MTX-naive RA}

In the phase III SELECT-EARLY trial, monotherapy with the selective JAK1 inhibitor upadacitinib was superior to methotrexate (MTX) in clinical, radiographic and patientreported outcomes in MTX-naive patients with active rheumatoid arthritis (RA).

The results support those of other pivotal phase III trials showing the therapeutic potential of upadacitinib in RA.

The SELECT-EARLY trial enrolled 947 patients from 43 countries who had moderately to severely active RA and had received no or minimal (three or fewer weekly doses) MTX. Most of the study population had early RA (median disease duration 0.5 years) and risk factors for structural progression, namely positivity for both rheumatoid factor and anti-citrullinated protein antibodies and/or at least one bone erosion. Patients were randomly allocated to receive once-daily upadacitinib (15 mg or $30 \mathrm{mg}$ ) or weekly MTX (7.5-20 mg per week) for 24 weeks.

The study met both of its primary end points. At week $12,52 \%$ of patients who received upadacitinib $15 \mathrm{mg}$ and $56 \%$ of those who received the $30 \mathrm{mg}$ dose achieved an ACR50 response, compared with $28 \%$ of those treated with MTX. The proportion of patients who achieved a DAS28-CRP score $<2.6$ at week 24 was also higher in the upadacitinib $15 \mathrm{mg}$ and $30 \mathrm{mg}$ groups ( $48 \%$ and $50 \%$, respectively) than in the methotrexate group (19\%). For both ACR50 response and DAS28-CRP score, the differences between each of the upadacitinib groups and the MTX group were apparent at week 2 and persisted through week 24.

Over 24 weeks, $88-89 \%$ of patients in the upadacitinib groups had no change from baseline in modified total Sharp score, indicating progression of structural damage was prevented. Greater improvements in patient-reported outcomes of fatigue, function and health-related quality of life were recorded in the upadacitinib groups than in the MTX group. The rate of adverse events was slightly higher in the upadacitinib $30 \mathrm{mg}$ group than the other groups.

Sarah Onuora

ORIGINAL ARTICLE van Vollenhoven, R. et al. Efficacy and safety of upadacitinib monotherapy in methotrexate-naïve patients with moderately to severely active rheumatoid arthritis (SELECT-EARLY): a randomized, double-blind, active-comparator, multi-center, multi-country trial. Arthritis Rheumatol. https://doi.org/10.1002/art.41384 (2020)

\title{
Microbiota, metabolism and lupus in mice
}

Changes in the gut microbiota have previously been associated with systemic lupus erythematosus (SLE) in humans and in mouse models of the disease, as have changes in the metabolism of tryptophan, which requires microbial involvement. However, a causative link between gut microbiota dysbiosis, tryptophan metabolism and autoimmune disease was lacking. In a new study published in Science Translational Medicine, researchers demonstrated that dysbiotic gut microbiota from lupus-prone mice can initiate autoimmunity in healthy mice and that this disease trigger can be modified by manipulating tryptophan metabolism.

Fecal transfer from B6.Sle1.Sle2.Sle3 lupus-prone mice to control C57BL/6 mice (which are $95 \%$ genetically identical to the lupus-prone mice) caused the control mice to develop autoimmunity, but only when the donor lupus-prone mice showed signs of autoimmune disease. However, co-housing of lupus-prone and control mice reduced autoimmunity in the lupus-prone mice but did not generate full-blown autoimmunity in the control mice, suggesting a mitigating effect of horizontal transfer of microbes.

Metabolism of tryptophan in lupus-prone mice was altered compared with control mice and could be restored to normal by antibiotic treatment. In addition, changing the amount of dietary tryptophan available to the mice altered the gut microbiota of both lupus-prone and control mice, indicating a firm link between the gut microbiota and tryptophan metabolism.

Extending this link to autoimmunity, fecal transfer from lupus-prone mice fed a high-tryptophan diet (which worsened their disease) to germ-free control mice induced autoimmunity, whereas fecal transfer from lupus-prone mice fed a low-tryptophan diet (which prevented disease) did not.

Further studies will be necessary to investigate whether gut microbiota dysbiosis and tryptophan metabolism have the same role in patients with SLE.

Joanna Clarke

ORIGINAL ARTICLE Choi, S.-C. et al. Gut microbiota dysbiosis and altered tryptophan catabolism contribute to autoimmunity in lupus-susceptible mice. Sci. Transl Med. 12, eaxx2220(2020)

\section{FIBROSIS}

\section{JAK inhibition for Dupuytren disease?}

Many fibrotic disorders involve immune and inflammatory processes that are promoted by various cytokines, including transforming growth factor $\beta$ (TGF $\beta$ ) and IL-13. New findings suggest that Dupuytren disease, a common fibrotic disorder, has immune and inflammatory components that can be inhibited by targeting the JAK-STAT pathway.

Using immunohistochemistry analysis, the researchers identified an enrichment of immune cells in tissue from patients with Dupuytren disease undergoing surgical fasciectomy compared with tissue from patients without Dupuytren disease undergoing hand surgery for carpal tunnel syndrome, including the presence of IL-13-producing mast cells and IFN $\gamma$-producing $T$ cells.

To further explore the potential consequences of a cytokine-rich environment, they performed various in vitro experiments on mast cells, fibroblasts from healthy carpal fascia tissue (control fibroblasts) and myofibroblasts from Dupuytren nodules. IFN $\gamma$ promoted the production of IL-13 by mast cells and, together with TGF $\beta$, increased the expression of the IL-13 receptor IL-13Ra1 by control fibroblasts. Both effects were accompanied by an increase in STAT1 signalling.

IL-13 treatment of control fibroblasts or Dupuytren myofibroblasts promoted cell proliferation, the production of extracellular matrix components (including type I collagen, tenascin C and periostin) and STAT6 signalling. Notably, these effects were greater in the Dupuytren myofibroblasts.

"Differential STAT1 binding at the IL-13R 1 gene locus drove the enhanced responsiveness of Dupuytren cells to IL-13 compared with control fibroblasts," explains Neal Millar, corresponding author on the study. "This finding is strongly suggestive of epigenetic modifications."

Most importantly, a JAK inhibitor (tofacitinib) could decrease STAT signalling in all three cell types and inhibited the fibroproliferative effects of IFN $\gamma$ and IL-13.

Jessica McHugh

ORIGINAL ARTICLE Akbar, M. et al. Attenuation of Dupuytren's fibrosis via targeting of the STAT1 modulated IL-13Ra1 response. Sci. Adv. 6, eaaz8272 (2020) 